\title{
Vehicle Reference Generator for Collision-Free Trajectories in Hazardous Maneuvers
}

\author{
Cuauhtémoc Acosta Lúa $\mathbb{D}^{1},{ }^{1}$ Gerardo De Jesus Díaz, \\ Stefano Di Gennaro (iD), ${ }^{2}$ and Tarek Kabbani ${ }^{2}$ \\ ${ }^{1}$ Departamento de Ciencias Tecnológicas, Centro Universitario de la Ciénega, Universidad de Guadalajara, Av. Universidad 1115, \\ 47820 Ocotlán, JAL, Mexico \\ ${ }^{2}$ Department of Information Engineering, Computer Science and Mathematics, Center of Excellence DEWS, University of L'Aquila, \\ Via Vetoio, Loc. Coppito, 67100 L'Aquila, Italy
}

Correspondence should be addressed to Cuauhtémoc Acosta Lúa; cuauhtemoc.acosta@cuci.udg.mx

Received 4 August 2017; Accepted 30 January 2018; Published 27 February 2018

Academic Editor: Gabriele Cazzulani

Copyright (c) 2018 Cuauhtémoc Acosta Lúa et al. This is an open access article distributed under the Creative Commons Attribution License, which permits unrestricted use, distribution, and reproduction in any medium, provided the original work is properly cited.

\begin{abstract}
This paper presents a reference generator for ground vehicles, based on potential fields adapted to the case of vehicular dynamics. The reference generator generates signals to be tracked by the vehicle, corresponding to a trajectory avoiding collisions with obstacles. This generator integrates artificial forces of potential fields of the object surrounding the vehicle. The reference generator is used with a controller to ensure the tracking of the accident-free reference. This approach can be used for vehicle autonomous driving or for active control of manned vehicles. Simulation results, presented for the autonomous driving, consider a scenario inspired by the so-called moose (or elk) test, with the presence of other collaborative vehicles.
\end{abstract}

\section{Introduction}

One important problem to be solved in the case of autonomous vehicle, as well as in active controlled vehicles, is the generation of proper reference signals ensuring the prevention from collisions with obstacles. The situation is rendered more articulated by the possible presence in the scenario of other collaborative and noncollaborative vehicles. In this paper, the artificial potential fields for autonomous robot guidance, first proposed in [1], is reformulated in the case of vehicular dynamics to determine a reference generator, which generates signals corresponding to a trajectory avoiding collisions with obstacles. Then a controller ensures the tracking of these reference signals.

In robotics, the generation of a reference using potential fields has been largely investigated. The basic idea is simple: obstacles generate repulsive forces, whereas goals to be reached generate attractive forces. Different approaches have been considered to have appropriate field configurations (e.g., see [2-4]) and handle moving obstacles and goals. Environmental hazards can be handled with simplicity and these methods can be implemented in real time, since the computation effort is not heavy [5].

Among the various techniques that can be used for reference generation, one can use the navigation function [6], the construction of an admissible trajectory space [7], or control and optimization techniques $[8,9]$. The latter use the model predictive control to generate the reference trajectory, neglecting the dynamic environment, and are usually complex and difficult to implement online. Other methods consider only indoor robots [10], whereas [11] proposes an algorithm based on graph searching and configuration space discretization to guide an indoor robot, but collision avoidance is not considered.

In this paper, a reference generator for ground vehicles, based on potential fields, is presented. The aim is the generation of trajectories avoiding collisions with obstacles. This is done by a reference generator, in which the forces due to the potentials associated with the obstacles and with the goals appear. These potential fields, similar to the barrier forces [12], are modified to the case of vehicular dynamics, adopting elliptic shapes of the potential fields, 
which adapt better to this environment. This adaptation allows having different force values for the different directions of the approaching obstacles. This makes this method more convenient in a dynamic environment such as the vehicular one. The reference generator represents a "reference vehicle" whose dynamics determine the reference to be tracked by the "real vehicle." The trajectories generated by this reference generator can be used for vehicle autonomous driving or for vehicle active control. A controller will ensure the tracking of the reference signals: in the case of autonomous vehicles, the control action is imposed by the control system; in the case of vehicles with human drivers, the control system intervenes (via active control devices; see, e.g., [13] and references therein) to correct the driver's action in order to correctly follow the references.

In addition to the adaptation of the artificial potential fields to the vehicular environment on a road, adopting elliptic shapes, the originality of this paper resides in the fact that the forces of the potential fields are applied to the reference generator rather than to the real vehicle, as commonly done in the literature [14-17]. The advantages to proceed in this way are that the obstacle avoidance problem is divided into two independent subproblems (reference generation and tracking problem, where this latter can be solved with any desired technique) and that the virtual forces may be also discontinuous (as in the case of obstacles that appear as event-driven dynamics: traffic lights, sudden external disturbance, etc.), whereas the resulting generated references are continuous. This latter aspect implies that the controller is not discontinuous and therefore can be physically implemented.

The proposed method is tested in a scenario inspired by the so-called moose (or elk) test, better standardized by the ISO $3888-2$ and here freely modified to consider also evasive maneuvers that generically consider also the presence of other collaborative vehicles, communicating via vehicle-to-vehicle $(\mathrm{V} 2 \mathrm{~V})$ communications. This test is quite compelling and significative of hazardous situations in the vehicular environment. The scenario has been implemented in Simulink as first step in the performance evaluation before testing this approach with more realistic vehicle dynamics software.

The paper is organized as follows. In Section 2, the mathematical model of a vehicle is recalled, and the control problem is stated. In Section 3, the reference generator with attractive and repulsive forces is introduced. In Section 4, the attractive and repulsive potential fields associated with obstacles and goals are proposed, and in Section 5 a controller ensuring reference tracking is designed. In Section 6, some simulations are provided, which show that the proposed method is effective in the scenario taken into account. Some comments and future work conclude the paper.

\section{Mathematical Model of a Ground Vehicle}

The attitude dynamics of a vehicle can be described by the dynamics of the longitudinal and lateral velocities $v_{x}$ and $v_{y}$

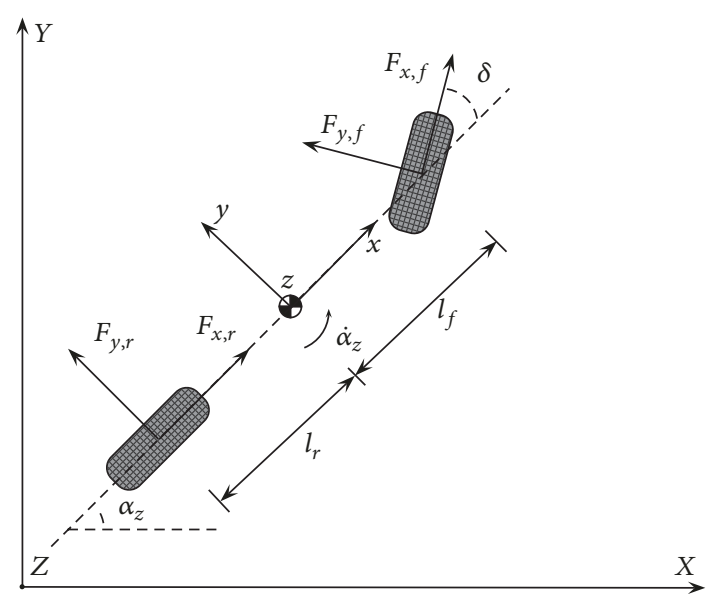

FIGURE 1: Schematic diagram of the bicycle model.

of the vehicle and by the dynamics of its yaw angular velocity $\omega_{z}:$

$$
\begin{aligned}
& \dot{v}_{x}=v_{y} \omega_{z}+\frac{\mu_{x}}{m}\left(F_{x, f}+F_{x, r}\right), \\
& \dot{v}_{y}=-v_{x} \omega_{z}+\frac{\mu_{y}}{m}\left(F_{y, f}+F_{y, r}\right), \\
& \dot{\omega}_{z}=\frac{\mu_{y}}{J_{z}}\left(F_{y, f} l_{f}-F_{y, r} l_{r}\right)+\frac{1}{J_{z}} M_{z} .
\end{aligned}
$$

This model, also called bicycle model or single-track model, even if simplified, well captures the essence of the dynamics to be controlled [18]. In (1), $m$ and $J_{z}$ are the vehicle mass and inertia momentum, $l_{f}$ and $l_{r}$ are the front and rear vehicle length, and $v_{x}$ and $v_{y}$ are the longitudinal and lateral velocities of the vehicle center of mass. Moreover, $\mu_{x}$ and $\mu_{y}$ are the longitudinal and lateral tire-road friction coefficient and $F_{x, f}$ and $F_{x, r}$ and $F_{y, f}$ and $F_{y, r}$ are the longitudinal and lateral forces due to the front and rear tires, normalized with respect to $\mu_{x}$ and $\mu_{y}$.

The state variables are expressed in the frame $R \Gamma(G, x, y, z)$, fixed with the vehicle and centered in its center of mass $G$, whereas the inertial frame is denoted as $R C(O, X, Y, Z)$ (see Figure 1).

The forces $F_{x, f}$ and $F_{x, r}$ influence the longitudinal dynamics, and the lateral forces $F_{y, f}$ and $F_{y, r}$ influence the lateral dynamics (but also the yaw dynamics). Finally, the torque $M_{z}$ influences the yaw dynamics. This latter torque can be realized by Rear Torque Vectoring (RTV) devices, that is, actuators capable of generating a torque on the vehicle, via braking or engine power differential distribution between the left and right vehicle sides (see, e.g., [19-23] for further details).

The front/rear lateral forces $F_{y, f}\left(\alpha_{f}\right)$ and $F_{y, r}\left(\alpha_{r}\right)$ depend on the front/rear tire slip angles $\alpha_{f}=\delta_{s}-\left(v_{y}+l_{f} \omega_{z}\right) / v_{x}$ and $\alpha_{r}=-\left(v_{y}-l_{r} \omega_{z}\right) / v_{x}$, with $\delta_{s}$ being the steering angle imposed at the front wheel [24]. The front/rear longitudinal forces $F_{x, f}\left(\lambda_{f}\right)$ and $F_{x, r}\left(\lambda_{r}\right)$ depend on the front/rear tire slips $\lambda_{f}=1-\omega_{w, f} R_{w} / v_{x}$ and $\lambda_{r}=1-\omega_{w, r} R_{w} / v_{x}$, with $\omega_{w, f}$ 
and $\omega_{w, r}$ being the front/rear wheel angular velocities and $R_{w}$ being the wheel radius [24].

The angle $\delta_{s}$ can be imposed by the driver or by the control system, in the case of manual or autonomous vehicle, or by both in the case of active control of the vehicle, with devices such as the Active Front Steering (AFS) and RTV, used to assist and correct the driver's actions [13]. In this last case, $\delta_{s}$ is the sum of two terms, one due to the driver and the other due to the AFS. In the following, the angle $\delta_{s}$ is assumed to be at least continuously differentiable with respect to time, which is a very mild hypothesis, verified in practice.

Finally, the dynamics of the wheels are

$$
\dot{\omega}_{w, j}=\frac{1}{J_{w, j}}\left(T_{e, j}-T_{b, j}-R_{w}\left(F_{t, j}+F_{w, j}\right)\right), \quad j=f, r,
$$

where $T_{e, j}$ and $T_{b, j}$ are the torques imposed by the engine and applied by the brake to the wheel $j=f, r$. Moreover, for the wheel $j=f, r, J_{w, j}$ is the wheel inertia, $F_{w, j}=\kappa_{j} \omega_{w, j}$ is the viscous friction force, $\kappa_{j}$ is the viscous friction coefficient, $F_{t, j}=\mu_{x, j} N=f\left(\lambda_{s, j}\right) m g$ is the traction force, $\mu_{x, j}=f\left(\lambda_{s, j}\right)$ is the adhesion coefficient, $N=m g$ is the normal load on the tire, and $g$ is the gravity acceleration. For the sake of simplicity and consistently with the single-track model (4), we consider that the front wheels have the same velocity and consider the same for the rear wheels.

In the literature, there are various models of tire (see, e.g., [24] and references therein). For the sake of simplicity, a simplified version of the Pacejka "magic formula" has been used in this work [24]:

$$
\begin{aligned}
& F_{y, j}\left(\alpha_{j}\right)=D_{y, j} \sin \left(C_{y, j} \arctan \left(B_{y, j} \alpha_{j}\right)\right), \\
& F_{x, j}\left(\lambda_{j}\right)=D_{x, j} \sin \left(C_{x, j} \arctan \left(B_{x, j} \lambda_{j}\right)\right),
\end{aligned}
$$

where $B_{i, j}, C_{i, j}$, and $D_{i, j}(i=x, y ; j=f, r)$ are experimental parameters. It is worth noting that the results of the paper can be applied also considering other tire models.

Model (1) is quite simple and nonlinear just for the terms $F_{x, f}, F_{x, r}, F_{y, f}$ and $F_{y, r}$. It can be used to design a reference generator and a control law to track such a reference. The control problem is to generate a collision-free path with reference velocities $v_{x \text {,ref }}, v_{y \text {,ref }}$, and $\omega_{z \text {,ref }}$ for the longitudinal, lateral, and angular velocity of (1) and a control law to track these references. To this aim, in the next section, a collision-free generator, making use of potential fields, will be introduced, and a control law will be designed to track these references.

\section{The Reference Generator with Attractive and Repulsive Forces}

The dynamics of the reference vehicle mimic the dynamics of a real vehicle:

$$
\begin{aligned}
\dot{v}_{x, \text { ref }}= & \omega_{z, \text { ref }} v_{y, \text { ref }}+\frac{\mu_{x, \text { ref }}}{m_{\text {ref }}}\left(F_{x, f, \text { ref }}+F_{x, r, \text { ref }}\right) \\
& +\frac{1}{m_{\text {ref }}} F_{t, x},
\end{aligned}
$$

$$
\begin{aligned}
\dot{v}_{y, \text { ref }}= & -\omega_{z, \text { ref }} v_{x, \text { ref }}+\frac{\mu_{y, \text { ref }}}{m_{\text {ref }}}\left(F_{y, f, \text { ref }}+F_{y, r, \text { ref }}\right) \\
& +\frac{1}{m_{\text {ref }}} F_{t, y}, \\
\dot{\omega}_{z, \text { ref }}= & \frac{\mu_{y, \text { ref }}}{J_{z, \text { ref }}}\left(F_{y, f, \text { ref }} l_{f}-F_{y, r, \text { ref }} l_{r}\right)+\frac{l_{p}}{J_{z, \text { ref }}} F_{t, y},
\end{aligned}
$$

with some notable differences:

(1) The forces $F_{x, f, \text { ref }}, F_{x, r, \text { ref }}, F_{y, f \text {,ref }}$, and $F_{y, r \text {,ref }}$ exerted by the tires of the reference vehicle are similar to those of the real vehicle (1) but correspond to a "nominal" tire and, in particular, $F_{y, r, \text { ref }}$ is modified so that no tailspins are possible in the reference vehicle. Usually, this is obtained ensuring that the rear tire characteristic is not decreasing after a certain value (corresponding to the maximal lateral force), as usually happens in a real tire.

(2) A virtual force $F_{t}$ is applied to the vehicle:

$$
F_{t}=\sum_{j=1}^{N_{r}} F_{r, j, \mathrm{ref}}+\sum_{i=1}^{N_{g}} F_{a, i, \mathrm{ref}}
$$

with components $F_{t, x}$ and $F_{t, y}$ in the reference frame $R \Gamma_{\text {ref }}\left(G_{\text {ref }}, x_{\text {ref }}, y_{\text {ref }}, z_{\text {ref }}\right)$ fixed with the reference vehicle. This force is the sum of the repulsive forces $F_{r, j, \text { ref }}$ due to the obstacles to be avoided and of the attractive forces $F_{a, i, \text { ref }}$ due to the goals to be reached. Among the obstacles to be avoided, there are also the path limits of the lane where the reference vehicle is travelling. These forces are applied to a point $P$, which in general does not coincide with the center of mass of the virtual vehicle and which may also be external to the geometric figure of the reference vehicle. $P$ can be chosen so that the virtual forces determine greater torques on the reference vehicle by augmenting the distance of $P$ from the center of mass in order to obtain better results in terms of steerability [25].

Moreover, $m_{\text {ref }}$ and $J_{z \text {,ref }}$ are the mass and inertia with respect to the $z$-axis ( $z$ is oriented so that $(x, y, z)$ is a right orthogonal frame), usually equal to the nominal values. Finally, $\mu_{x, \text { ref }}$ and $\mu_{y \text {,ref }}$ are the reference tire-road friction coefficients in the $x$ and $y$ directions, and $l_{f}$ and $l_{r}$ are the distances from the vehicle center of gravity $G_{\text {ref }}$ to the front and rear axles.

In Figure 2, a typical case of the reference vehicle in the presence of an obstacle and of a goal is shown, with the corresponding repulsive and attractive forces. The resulting force $F_{t}$ determines further steering of the reference vehicle, which adds to the steering due to the tire forces.

Once the reference signals (4) are generated, the "real vehicle" (1) will be controlled in order to follow these references.

\section{Attractive and Repulsive Potential Fields}

An attractive potential field can be associated with a goal that one wants to reach. Analogously, a repulsive potential 


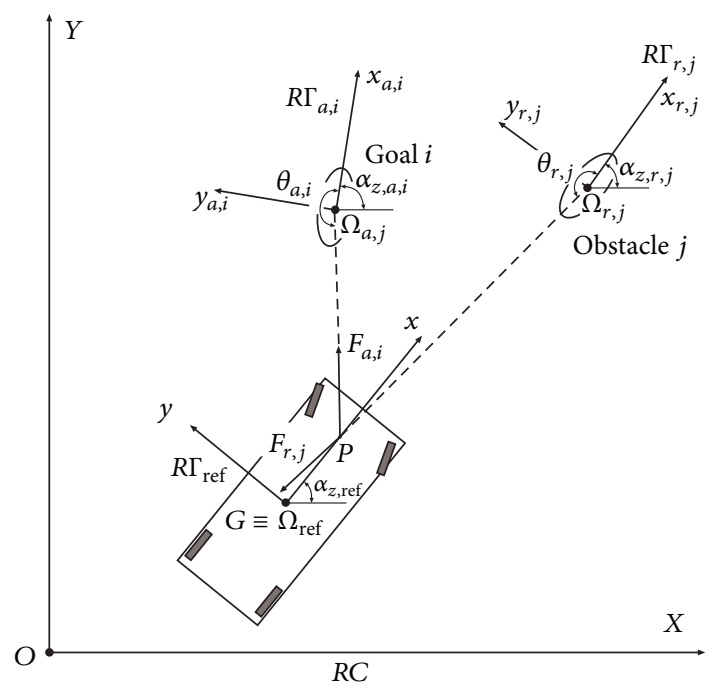

Figure 2: Reference vehicle, inertial and reference frames $R C$ and $R \Gamma_{\text {ref }}$, attractive force $F_{a, i}$ due to the $i^{\text {th }}$ goal, and repulsive force $F_{r, i}$ due to the $j^{\text {th }}$ obstacle.

field can be associated with an obstacle to be avoided. These potential fields determine attractive or repulsive forces acting, as already explained, on the reference vehicle. These potential fields have a maximal space in which they can act. Moreover, there is a space in which the reference vehicle cannot enter and corresponds to a security distance to be respected. In general, one can consider $N_{g}$ goals and $N_{r}$ obstacles, which may be moving.

Flags can be associated with specific maneuvers, which can be decided by a higher-level decision layer. In this work, flags $\mathscr{F}_{o}$ and $\mathscr{F}_{b}$ are associated with the overtaking and braking maneuvers with $\mathscr{F}_{o}=1$ when the vehicle is overtaking and zero otherwise. It is likewise for the braking maneuver flag $\mathscr{F}_{b}$. The use of these flags allows omitting in the reference vehicle (4) some forces that have not been considered in the maneuver, therefore improving the behavior of the reference vehicle during its maneuvers. Further flags can be used to omit repulsive forces of other vehicles that, via $\mathrm{V} 2 \mathrm{~V}$ communications, transmit their collaboration to avoid collisions to the moving vehicle.

4.1. Repulsive Potential Field due to an Obstacle. The repulsive potential field associated with an obstacle $j$ is given by

$$
\begin{aligned}
& U_{r, j} \\
& = \begin{cases}\text { undefined } & \text { if } \rho_{r, j}<s_{r, j} \\
\frac{\eta_{r, j}\left(v_{r, \text { rel }, j}\right)}{2} \frac{1}{s_{r, j}} \ln \frac{\left(\rho_{r, j}+s_{r, j}\right)^{r_{r, j}+s_{r, j}}}{\left(\rho_{r, j}-s_{r, j}\right)^{r_{r, j}-s_{r, j}}} & \text { if } \rho_{r, j} \in\left[s_{r, j}, r_{r, j}\right] \\
0 & \text { otherwise, }\end{cases} \\
& j=1, \ldots, N_{r} \text {, where } \\
& s_{r, j}\left(\theta_{r, j},\left|v_{r, \mathrm{rel}, j}\right|\right)=\frac{1}{\sqrt{a_{s, r, j}^{2} \cos ^{2} \theta_{r, j}+b_{s, r, j}^{2} \sin ^{2} \theta_{r, j}}},
\end{aligned}
$$

$$
r_{r, j}\left(\theta_{r, j}\right)=\frac{1}{\sqrt{a_{r, r, j}^{2} \cos ^{2} \theta_{r, j}+b_{r, r, j}^{2} \sin ^{2} \theta_{r, j}}}
$$

are the (elliptic) safety distance from the $j^{\text {th }}$ obstacle and the (elliptic) influence range of the potential field, respectively, with $\theta_{r, j}$ being the bearing angle. For practical reasons, the ellipse axis $a_{s, r, j}>b_{s, r, j}>0$ is chosen as

$$
\begin{aligned}
& a_{s, r, j} \\
& = \begin{cases}\frac{1}{v_{r, 1}} & \text { if } \gamma_{s, r, j}\left(\left|v_{r, \text { rel }, j}\right|\right) \in\left[0, v_{r, 1}\right) \\
\frac{1}{\left(\gamma_{s, r, j}\left(\left|v_{r, \text { rel }, j}\right|\right)\right)} & \text { if } \gamma_{s, r, j}\left(\left|v_{r, \text { rel }, j}\right|\right) \in\left[v_{r, 1}, v_{r, 2}\right] \\
\frac{1}{v_{r, 2}} & \text { if } \gamma_{s, r, j}\left(\left|v_{r, \text { rel }, j}\right|\right)>v_{r, 2},\end{cases}
\end{aligned}
$$

where $\gamma_{s, r, j}\left(\left|v_{r, \text { rel }, j}\right|\right)$ is the major axis gain, which increases $s_{r, j}$ according to the velocity intensity $\left|v_{r, \text { rel }, j}\right|$. For instance, one can take $\gamma_{s, r, j}\left(\left|v_{r, \text { rel }, j}\right|\right)=\gamma_{s, r, j}^{\circ}\left|v_{r, \text { rel }, j}\right|$, with $v_{r, 2}>v_{r, 1}>$ $1 / b_{s, r, j}>0$ and $\gamma_{s, r, j}^{\circ}>0$. Moreover, the ellipse axes of the influence region are such that $a_{r, r, j}>b_{r, r, j}>0$, so that $a_{r, r, j}>$ $a_{s, r, j}$ and $b_{r, r, j}>b_{s, r, j}$. In this way, $r_{r, j}>s_{r, j}$. Since $s_{r, j}$ depends on $v_{r, \text { rel, } j}$, the safety and the influence regions have different shapes in general.

The potential filed contains a logarithmic term to have a steeper increase of the repulsive force when $\rho_{r, j}$ tends to the safety distance $s_{r, j}$. The gains $\eta_{r, j}$, which can be generically different for each obstacle, are (e.g., linear or quadratic) functions of the relative velocity $v_{r, \text { rel, }, j}$, expressed in $R \Gamma_{r, j}$ fixed with the obstacle, between the velocity of the $j^{\text {th }}$ obstacle and that of the reference vehicle. The dependence on $v_{r, \text { rel }, j}$ allows taking into account the different situations that can occur in practical situations. Moreover,

$$
\rho_{r, j}=\sqrt{\left(x_{P}-x_{j}\right)^{2}+\left(y_{P}-y_{j}\right)^{2}}
$$

is the distance of the point $P$ of the reference vehicle from the $j^{\text {th }}$ obstacle; $\left(x_{j}, y_{j}, 0\right)$ are the coordinates of origin $\Omega_{r, j}$ of the reference frame $R \Gamma_{r, j}\left(\Omega_{r, j}, x_{r, j}, y_{r, j}, z_{r, j}\right)$ fixed with the $j^{\text {th }}$ obstacle (usually $\Omega_{r, j}$ coincides with the geometric center of the obstacle), expressed in the frame $R \Gamma_{\text {ref }}$ fixed with the reference vehicle. Note that, for a fixed distance $\rho_{r, j}$, the potential field varies with the bearing angle $\theta_{r, j}$, so that one gets higher repulsive forces when $\theta_{r, j}=0$, that is, when the reference vehicle is following the obstacle, and lower values when $\theta_{r, j}=\pi / 2$, that is, when the reference vehicle is avoiding the obstacle.

The resulting repulsive force exerted on the reference vehicle, due to the potential field $U_{r, j}$ in (6), has components in $R \Gamma_{r, j}$ given by

$$
F_{r, j}=-\frac{\partial U_{r, j}}{\partial \rho_{r, j}}\left(\begin{array}{c}
\cos \theta_{r, j} \\
\sin \theta_{r, j}
\end{array}\right)=\varphi_{j}\left(\rho_{r, j}, \theta_{r, j}\right)\left(\begin{array}{c}
\cos \theta_{r, j} \\
\sin \theta_{r, j}
\end{array}\right),
$$




$$
\begin{aligned}
& \varphi_{j}\left(\rho_{r, j}, \theta_{r, j}\right) \\
& = \begin{cases}\text { undefined } & \text { if } \rho_{r, j}<s_{r, j} \\
-\eta_{r, j}\left(v_{r, \text { rel }, j}\right) \frac{\rho_{r, j}-r_{r, j}\left(\theta_{r, j}\right)}{\rho_{r, j}^{2}-s_{r, j}^{2}\left(\theta_{r, j}\right)} & \text { if } \rho_{r, j} \in\left[s_{r, j}, r_{r, j}\right] \\
0 & \text { otherwise, }\end{cases}
\end{aligned}
$$

$j=1, \ldots, N_{r}$. This force expressed in the reference $R \Gamma_{\text {ref }}$ is

$$
F_{r, j \text {,ref }}=\mathscr{F} \mathscr{R}_{\text {ref }, \text { in }}\left(\alpha_{z, \text { ref }}\right) \mathscr{R}_{r, j, \text { in }}^{T}\left(\alpha_{z, r, j}\right) F_{r, j},
$$

where $\mathscr{F}=\operatorname{diag}\left\{\mathscr{F}_{b}, \mathscr{F}_{o}\right\}$ is a flag matrix and

$$
\begin{aligned}
& \mathscr{R}_{\text {ref }, \text { in }}\left(\alpha_{z, \text { ref }}\right)=\left(\begin{array}{cc}
\cos \alpha_{z, \text { ref }} & \sin \alpha_{z, \text { ref }} \\
-\sin \alpha_{z, \text { ref }} & \cos \alpha_{z, \text { ref }}
\end{array}\right), \\
& \mathscr{R}_{r, j \text {,in }}\left(\alpha_{z, r, j}\right)=\left(\begin{array}{cc}
\cos \alpha_{z, r, j} & \sin \alpha_{z, r, j} \\
-\sin \alpha_{z, r, j} & \cos \alpha_{z, r, j}
\end{array}\right)
\end{aligned}
$$

are the rotation matrices that transform, respectively, vectors expressed in $R C$ into vectors expressed in $R \Gamma_{\text {ref }}$ and vectors expressed in $R C$ into vectors expressed in $R \Gamma_{\mathrm{r}, j}$. Here $\alpha_{z, \text { ref }}$ and $\alpha_{z, r, j}$ are the yaw angles of the reference vehicle and of the obstacle, respectively.

4.2. Attractive Potential Field due to a Goal. The potential field associated with a goal $i$ is given by

$$
\begin{aligned}
& U_{a, i} \\
& = \begin{cases}\text { undefined } & \text { if } \rho_{a, i}<s_{a, i} \\
-\frac{\eta_{a, i}\left(v_{a, \text { rel }, i}\right)}{2} \frac{1}{s_{a, i}} \ln \frac{\left(\rho_{a, i}+s_{a, i}\right)^{r_{a, i}+s_{a, i}}}{\left(\rho_{a, i}-s_{a, i}\right)^{r_{a, i}-s_{a, i}}} & \text { if } \rho_{a, i} \in\left[s_{a, i}, r_{a, i}\right] \\
0 & \text { otherwise, }\end{cases}
\end{aligned}
$$

where $\eta_{a, i}$ is the potential field gain (e.g., a linear or a quadratic function of the relative velocity $v_{a, \text { rel }, i}$ between the velocity of the $i^{\text {th }}$ goal and that of the reference vehicle, again to take into account the different situations that can occur in practical situations), $\rho_{a, i}=\sqrt{\left(x_{P}-x_{i}\right)^{2}+\left(y_{P}-y_{i}\right)^{2}}$ is the distance from the goal, and $\left(x_{i}, y_{i}, 0\right)$ are the coordinates of the origin $\Omega_{a, i}$ expressed in $R \Gamma_{\text {ref }}$;

$$
\begin{aligned}
& s_{a, i}\left(\theta_{a, i}\right)=\frac{1}{\sqrt{a_{s, a, i}^{2} \cos ^{2} \theta_{a, i}+b_{s, a, i}^{2} \sin ^{2} \theta_{a, i}}}, \\
& r_{a, i}\left(\theta_{a, i}\right)=\frac{1}{\sqrt{a_{r, a, i}^{2} \cos ^{2} \theta_{a, i}+b_{r, a, i}^{2} \sin ^{2} \theta_{a, i}}}
\end{aligned}
$$

are the (elliptic) safety distance to maintain when following the goal and the (elliptic) influence range of the goal, respectively, with $\theta_{a, i}$ being the goal bearing angle. Here, $a_{s, a, i}>b_{s, a, i}>0$, and $a_{s, a, i}$ has an expression similar to (8). Finally, $a_{r, a, i}>b_{r, a, i}>0$ so that $a_{r, a, i}>a_{s, a, i}, b_{r, a, i}>b_{s, a, i}$, and $r_{a, i}>s_{a, i}$.
The force exerted on the reference vehicle expressed in $R \Gamma_{\text {ref }}$ is given by

$$
F_{a, i, \text { ref }}=\mathscr{R}_{\text {ref }, \text { in }}\left(\alpha_{z, \text { ref }}\right) \mathscr{R}_{a, i, \text { in }}^{T}\left(\alpha_{z, a, i}\right) F_{a, i},
$$

$i=1, \ldots, N_{g}$, where

$$
\begin{aligned}
& F_{a, i}=-\frac{\partial U_{a, i}}{\partial \rho_{a, i}}\left(\begin{array}{c}
\cos \theta_{a, i} \\
\sin \theta_{a, i}
\end{array}\right)=\varphi_{i}\left(\rho_{a, i}, \theta_{a, i}\right)\left(\begin{array}{c}
\cos \theta_{a, i} \\
\sin \theta_{a, i}
\end{array}\right), \\
& \varphi_{i}\left(\rho_{a, i}, \theta_{a, i}\right) \\
& = \begin{cases}\text { undefined } & \text { if } \rho_{a, i}<s_{a, i} \\
\eta_{a, i}\left(v_{a, \mathrm{rel}, i}\right) \frac{\rho_{a, i}-r_{a, i}\left(\theta_{a, i}\right)}{\rho_{a, i}^{2}-s_{a, i}^{2}\left(\theta_{a, i}\right)} & \text { if } \rho_{a, i} \in\left[s_{a, i}, r_{a, i}\right] \\
0 & \text { otherwise }\end{cases}
\end{aligned}
$$

and $\mathscr{R}_{a, i, \text { in }}\left(\alpha_{z, a, i}\right)$ is the rotation matrix from $R C$ to $R \Gamma_{a, i}$, with $\alpha_{z, a, i}$ being the yaw angle of $R \Gamma_{a, i}$ with respect to $R C$.

\section{A Tracking Controller of the Reference Vehicle}

A simple controller is designed for the vehicle dynamics (1) to track the reference generated by (4), considering the following Lyapunov candidate [26]:

$$
V=\frac{1}{2} e_{v_{x}}^{2}+\frac{1}{2} e_{v_{y}}^{2}+\frac{1}{2} e_{\omega_{z}}^{2},
$$

where $e_{v_{x}}=v_{x}-v_{x, \text { ref }}, e_{v_{y}}=v_{y}-v_{y, \text { ref }}$, and $e_{\omega_{z}}=\omega_{z}-\omega_{z, \text { ref }}$. Using (1), its derivative is

$$
\begin{aligned}
\dot{V}= & e_{v_{x}}\left(v_{y} \omega_{z}+\frac{\mu_{x}}{m}\left(F_{x, f}+F_{x, r}\right)-\dot{v}_{x, \text { ref }}\right) \\
& +e_{v_{y}}\left(-v_{x} \omega_{z}+\frac{\mu_{y}}{m}\left(F_{y, f}+F_{y, r}\right)-\dot{v}_{y, \text { ref }}\right) \\
& +e_{\omega_{z}}\left(\frac{\mu_{y}}{J_{z}}\left(F_{y, f} l_{f}-F_{y, r} l_{r}\right)+\frac{1}{J_{z}} M_{z}-\dot{\omega}_{z, \text { ref }}\right),
\end{aligned}
$$

so that, considering

$$
\begin{aligned}
F_{x, f}+F_{x, r}= & \frac{m}{\mu_{x}}\left(-v_{y} \omega_{z}+\dot{v}_{x, \mathrm{ref}}-k_{1}\left(v_{x}-v_{x, \mathrm{ref}}\right)\right), \\
F_{y, f}= & -F_{y, r} \\
& +\frac{m}{\mu_{y}}\left(v_{x} \omega_{z}+\dot{v}_{y, \mathrm{ref}}-k_{2}\left(v_{y}-v_{y, \text { ref }}\right)\right), \\
M_{z}= & -\mu_{y}\left(F_{y, f} l_{f}-F_{y, r} l_{r}\right) \\
& +J_{z}\left(\dot{\omega}_{z, \mathrm{ref}}-k_{3} e_{\omega_{z}}\right),
\end{aligned}
$$

$k_{1}, k_{2}, k_{3}>0$, one finally works out

$$
\dot{V} \leq-k_{1} e_{v_{x}}^{2}-k_{2} e_{v_{y}}^{2}-k_{3} e_{\omega_{z}}^{2} .
$$


TABLE 1: Parameters of the reference and real vehicles and of the controller.

\begin{tabular}{lccc}
\hline$m_{\text {ref }}=m=1862 \mathrm{~kg}$ & $J_{z, \text { ref }}=J_{z}=2488 \mathrm{~kg} / \mathrm{m}^{2}$ & $l_{f, \text { ref }}=l_{f}=1.18 \mathrm{~m}$ & $k_{1}=35$ \\
$\mu_{x, \text { ref }}=\mu_{x}=1$ & $\mu_{y, \text { ref }}=\mu_{y}=1$ & $l_{r, \text { ref }}=l_{r, r}=1.77 \mathrm{~m}$ & $k_{2}=5$ \\
$\lambda_{s, \text { ref }}^{a}=\lambda_{s}^{a}=0.12$ & $\lambda_{s, \text { ref }}^{d}=\lambda_{s}^{d}=-0.12$ & $g=9.81 \mathrm{~m} / \mathrm{s}^{2}$ & $k_{3}=40$ \\
\hline
\end{tabular}

This proves the exponential tracking of the references. Note that (19) with (4) constitutes a dynamic controller.

Once the control actions (19) are determined, the forces $F_{x, f}, F_{x, r}, F_{y, f}$, and $M_{z}$ have to be physically implemented. In particular, regarding the lateral force $F_{y, f}$, since it is invertible with respect to $\alpha_{f}$ in the interval $\left[-\alpha_{f, \max }, \alpha_{f, \max }\right]$, where $\pm \alpha_{f, \max }$ are the slip angle value corresponding to the maximum and minimum of $F_{y, f}$, one can calculate the angle $\delta_{s}$ from (19). In fact, for a fixed value $F^{\circ}$, the solution of $F_{y, f}\left(\alpha_{f}\right)=F^{\circ}$ is unique and is given by

$$
\begin{aligned}
& \delta_{s} \\
& = \begin{cases}\frac{v_{y}+l_{f} \omega_{z}}{v_{x}}+F_{y, f}^{-1}\left(F^{\circ}\right) & \text { if } \alpha_{f} \in\left[-\alpha_{f, \max }, \alpha_{f, \max }\right] \\
\frac{v_{y}+l_{f} \omega_{z}}{v_{x}} \pm \alpha_{f, \max } & \text { otherwise. }\end{cases}
\end{aligned}
$$

The angle $\delta_{s}$ is the angle at the wheel which has to be imposed. When dealing with autonomous driving, $\delta_{s}$ is imposed by the control system of the vehicle. If the vehicle has a human driver who imposes a steering angle $\delta_{d}$, the active control system will impose an extra angle $\delta_{c}=\delta_{s}-\delta_{d}$ to on wheel in order to correctly follow the reference signals. The torque $M_{z}$ can be physically applied to the vehicle using differential braking between the left and right wheels (usually the rear wheels in order to be less invasive) or by means of other mechanisms, such as active differentials. Finally, as already noted, the forces $F_{x, f}$ and $F_{x, r}$ depend on the wheel slips $\lambda_{f}$ and $\lambda_{r}$. Denoting by $F_{x, f}^{\circ}$ and $F_{x, r}^{\circ}$ the values to be imposed at time $t^{\circ}$, let $\lambda_{f}^{\circ}$ and $\lambda_{r}^{\circ}$ be the corresponding slip values, obtained inverting the tires characteristics (3). They can be imposed by controlling the wheel slips [27]. To this aim, the values $\omega_{w, f}^{\circ}=\left(1-\lambda_{f}^{\circ}\right) v_{x} / R_{w}$ and $\omega_{w, r}^{\circ}=\left(1-\lambda_{r}^{\circ}\right) v_{x} / R_{w}$ are obtained and are imposed by means of $T_{e, f}$ and $T_{b, f}$ and $T_{e, r}$ and $T_{b, r}$ in (2), that is, controlling the velocities $\omega_{w, f}$ and $\omega_{w, r}$ of the front and rear wheels.

\section{Simulation Results}

In this section, a test has been considered to show the effectiveness of the proposed collision-free generator, inspired by the so-called moose (or elk) test, which is standardized by the ISO 3888-2. The scenario has been implemented in Simulink, for a first evaluation of the performance, before testing the dynamic controller (see (4) and (19)) in more realistic vehicle dynamics software. The scenario consists of a vehicle proceeding on a curving road, with a moose that suddenly crosses the road. A second vehicle (obstacle) proceeds in the opposite direction and can or cannot exchange data with the
TABLE 2: Parameters of the potential fields.

\begin{tabular}{lclc}
\hline$\eta_{r}^{\circ}=6.5 \times 10^{3}$ & $a_{r, r}=1 / 50$ & $b_{r, r}=1 / 4$ & $b_{s, r}=1 / 2$ \\
$v_{r, 1}=4$ & $v_{r, 2}=50$ & $\gamma_{s, r}^{\circ}=1.8$ & $\mu_{b}=0.9$ \\
\hline
\end{tabular}

first vehicle in order to have or not a collaborative action. See Figures 3 and 4 for further details on the scenario.

To complete the scenario description, we consider the case of autonomous driving (where $\delta_{d}=0$ ). The parameters values for the reference and real vehicles and for the controller are given in Table 1, whereas those used for generating the potential fields are in Table 2. The repulsive gain has been set equal to $\eta_{r}\left(v_{r, \text { rel }}\right)=\eta_{r}^{\circ}\left|v_{r \text {,rel }}\right|$, with $\eta_{r}^{\circ}$ determined experimentally.

The high-level controller of the vehicle can decide to brake or to avoid the moose. In the first case, the control system has to calculate the braking distance $s_{b}=v_{\text {ref }}^{2} /\left(2 \mu_{b} g\right)$ necessary to dissipate all vehicle kinetic energy and to stop before a collision, with $\mu_{b}$ being the dissipation coefficient, and $g$ is the acceleration constant. The high-level controller decides that a braking maneuver has to be carried out by the reference generator, setting $\mathscr{F}_{b}=1$, when the braking distance is greater than the distance $s_{0}$ with the obstacle (set equal to $15 \mathrm{~m}$ in the simulations). Otherwise, overtaking, that is, an evasive maneuver, is decided in order to avoid the moose, setting $\mathscr{F}_{o}=1$, if a vehicle-to-vehicle communication with the vehicle proceeding in the opposite direction gives its availability to brake or accelerate in order to ensure the existence of a proper temporal window for the evasive maneuver.

Figure 3 shows the case of a braking maneuver, where $s_{b}<s_{0}$, for avoiding the moose. The repulsive potential, centered on the moose, and the constraints due to the road allow generating a reference path (see Figure 3(a)), where the reference and real paths during the maneuver have been shown. The controller (19) follows perfectly the references generated by (4). In Figure 3(b), it is shown that the longitudinal velocity goes to zero, so that the distance $\rho_{r}$ remains greater than the safety distance $s_{a}$ (see Figure $3(\mathrm{~d})$ ). The generated longitudinal potential force that acts on the reference vehicle during braking (when $\rho_{r}$ is smaller than the influence range $r_{r}$ ) is shown in Figure $3(\mathrm{c})$.

In the second case, it is considered that $s_{b}>s_{0}$, and it is assumed that the vehicle proceeding in the opposite direction collaborates in order to allow the evasive maneuver (see Figure 4). Figures 4(b), 4(c), and 4(d) show the longitudinal, lateral, and angular velocities with values almost identical to the generated references. The repulsive (Figures 5(a) and 5 (b)) potential forces, acting on the reference vehicle, modify its dynamics.

It can be noticed from Figure 3(c) that when the distance $\rho_{r}$ between the reference vehicle and the obstacle is in the 


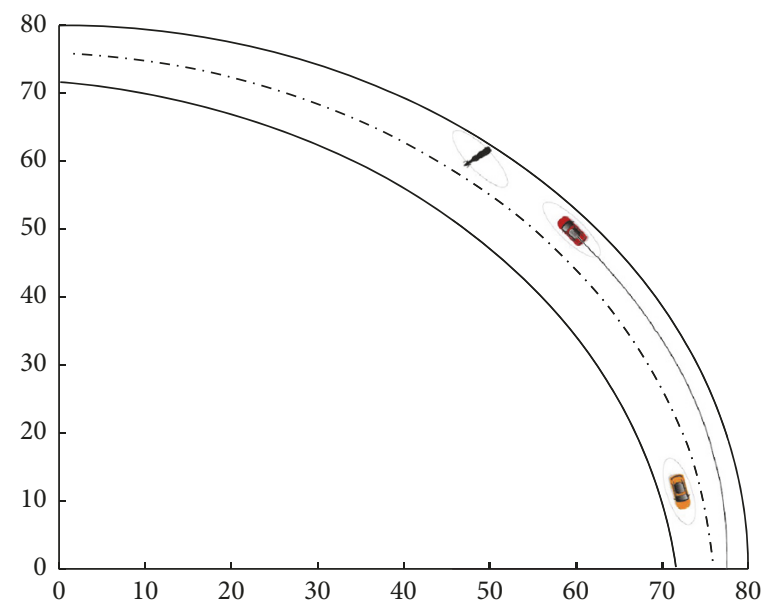

(a)

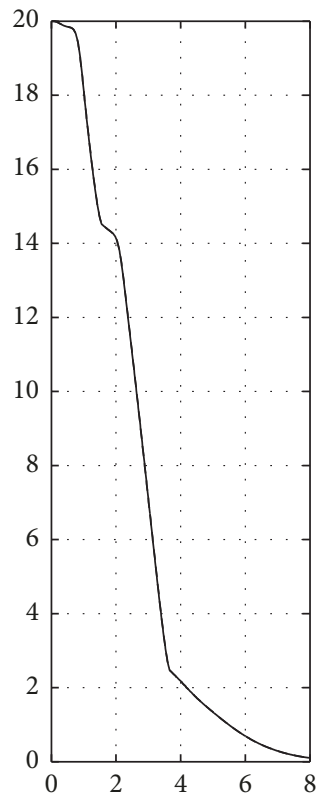

(b)

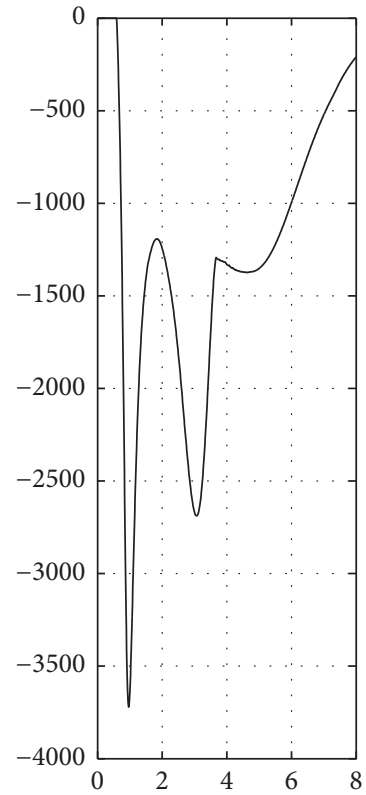

(c)

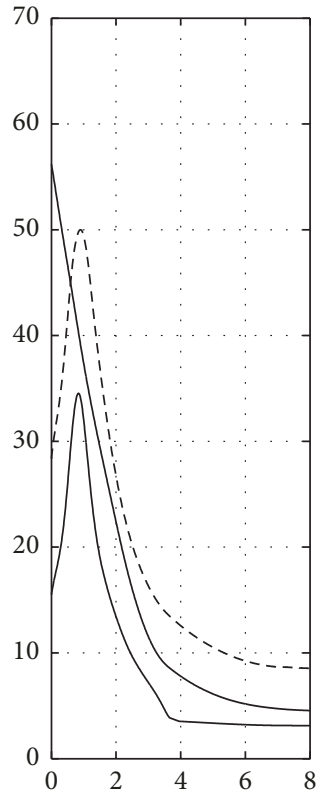

(d)

FiguRE 3: Braking maneuver before reaching the obstacle due to a moose. (a) Real (solid) and reference (dashed) trajectories. (b) Longitudinal velocity $v_{x}$ (black solid); reference $v_{x, \text { ref }}$ (black dashed) [m/s versus s]. (c) Repulsive force $F_{r, x}$ [N versus s]. (d) Distance $\rho_{r}$ between reference vehicle and the moose (black), influence distance $r_{r}$ (gray dashed), and safety distance $s_{r}$ (gray solid) [m versus s].

influence range $r_{r}$ of the repulsive potential field of the obstacle, the reference vehicle is affected by these repulsive forces. Note that $\rho_{r}$ remains, as expected, greater than the safety distance $s_{r}$. A temporal window allows the reference vehicle to perform the evasive maneuver. It should be noted that, due to the definition of the potential fields, the reference vehicle is not affected by the obstacle traveling in the opposite direction.

It is also important to note that as the potential fields does not implement any logic regarding the direction of the evasive maneuver and if the moose was closer to the middle of the road instead of being on the right lane, in this particular curved road, repulsion forces could be generated to make the evasive maneuver to the right of the moose. In this case, the evasive maneuver could not be accomplished successfully, leading to a collision with either the moose or the lane edge. Therefore, a further logic seems necessary to be added to the reference generator to choose the correct direction of the evasive maneuver or to choose a braking maneuver in order to mitigate the collision. This logic has to take into consideration some aspects concerning the context of the collision to avoid, such as the road topology, the heading of the obstacle with respect to the vehicle heading, and the direction of the available trajectory to which the evasive maneuver should be made.

Finally, it is worth noticing that both cases (braking and evasive maneuver) have to be intended as maneuvers to avoid the obstacle. In this sense, if $s_{b}>s_{0}$ and the evasive maneuver is not possible since another noncollaborative vehicle is approaching in the opposite lane, a collision is inevitable 


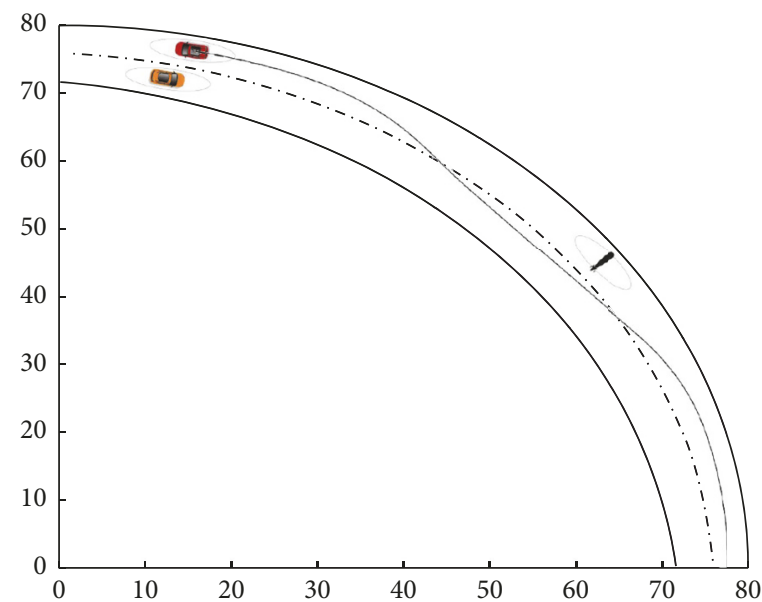

(a)

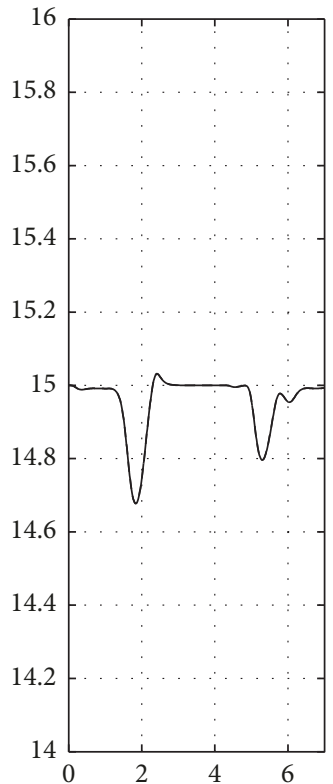

(b)

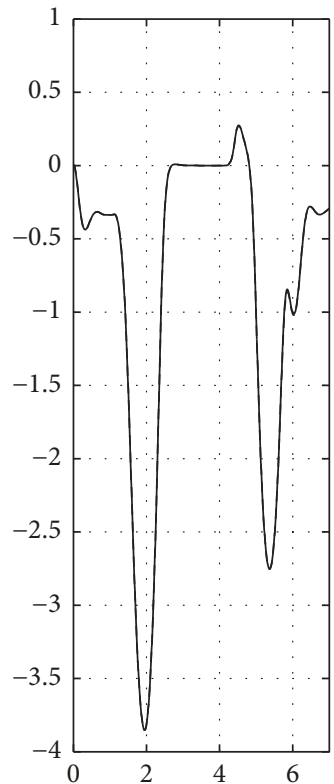

(c)

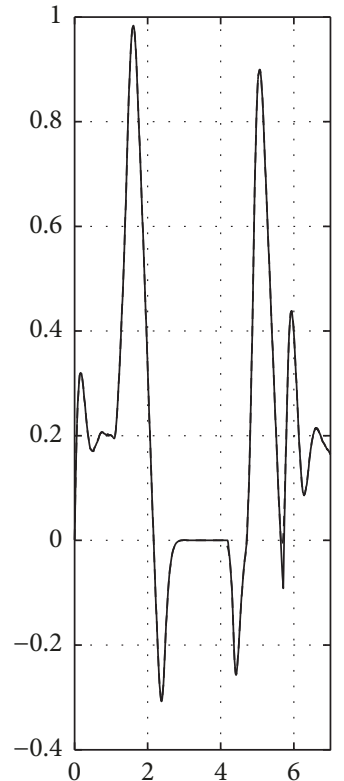

(d)

FIGURE 4: Evasive maneuver for avoiding the moose, with collaborating vehicle proceeding in the opposite lane. (a) Real (solid) and reference (dashed) trajectories. (b) Longitudinal velocity $v_{x}$ (black solid); reference $v_{x, \text { ref }}$ (black dashed) [m/s versus s]. (c) Lateral velocity $v_{y}$ (solid) and reference $v_{y, \text { ref }}$ (dashed) [m/s versus s]. (d) Angular velocities $\omega_{z}$ (solid) and reference $\omega_{z, \text { ref }}$ (dashed) $[\mathrm{rad} / \mathrm{s}$ versus s].

TABLE 3: Computational times.

\begin{tabular}{lllll}
\hline$T_{r}=0.06 \mathrm{~ms}$ & $T_{c}=0.05 \mathrm{~ms}$ & $T_{v}=0.03 \mathrm{~ms}$ & $T_{F_{r}}=0.02 \mathrm{~ms}$ & $T_{F_{a}}=0.02 \mathrm{~ms}$ \\
\hline
\end{tabular}

and the braking can be interpreted as a way to mitigate the collision effects.

A final remark deserves the implementability of the proposed method on commercial Electronic Control Units (ECUs). As first measure of implementability, we have proceeded to determine the computational time calculated at each cycle as the sum $T_{p}+T_{r}+T_{c}+T_{v}$, where $T_{r}, T_{c}$, and $T_{v}$ are the times required to calculate the reference, the control, and the dynamics of the real vehicle, respectively. Furthermore, $T_{p}$ is the computational time of the potential forces applied on the reference vehicle, which can be parameterized in terms of the $N_{r}$ obstacles and the $N_{g}$ goals: $T_{p}=N_{r} T_{F_{r}}+N_{g} T_{F_{a}}$, where $T_{F_{r}}$ and $T_{F_{a}}$ are the times required to calculate the repulsive force for one obstacle and the attractive force for one goal. Table 3 shows the calculation times obtained for an Intel Core ${ }^{\mathrm{TM}}$ Duo i5-3230M CPU@2.60 GHz HP PC, with 8 G of RAM and clock speed $2601 \mathrm{MHz}$. It can be seen that, for each cycle, the reference generator and the controller require $0.18 \mathrm{~ms}$ if $N_{r}=1$ and $N_{a}=1$. In the case considered in the simulations with $N_{r}=3$ and no goals, for each cycle, $0.2 \mathrm{~ms}$ 


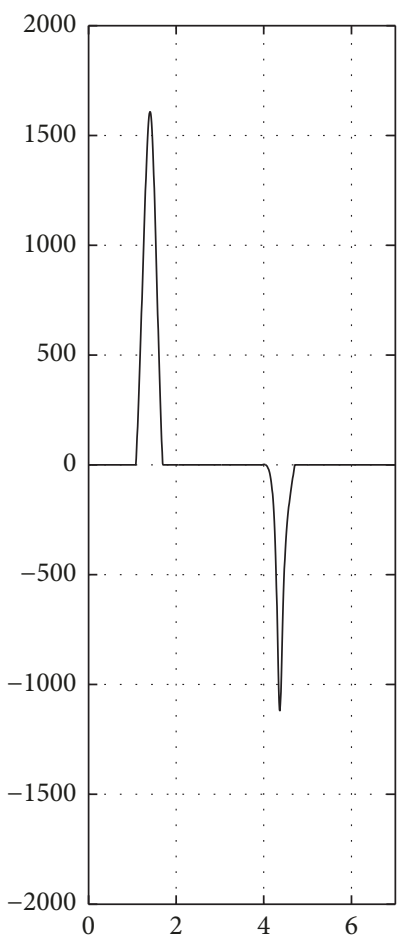

(a)

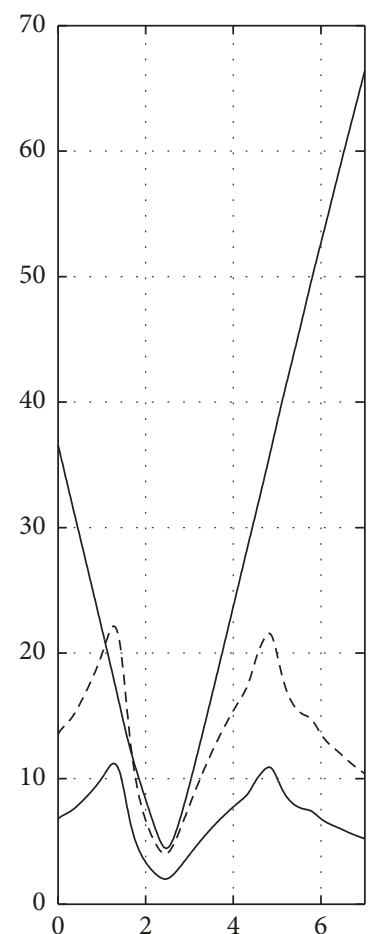

(b)

FIGURE 5: Evasive maneuver for avoiding the moose, with collaborating vehicle proceeding in the opposite lane. (a) Obstacle (moose) repulsive force $F_{r, y}$ [N versus s]. (b) Distance $\rho_{r}$ between reference and the moose (black), influence distance $r_{r}$ (gray dashed), and safety distance $s_{r}$ (gray solid) [m versus $\mathrm{s}$.

is required, whereas this time becomes $0.36 \mathrm{~ms}$ for $N_{r}=10$ and $N_{g}=1$. Therefore, the proposed technique appears to be implementable on a commercial ECU, where typical computational times are of the order of $10 \mathrm{~ms}$, as discussed in [28].

\section{Conclusions}

In this paper, the approach with potential fields has been adapted to the case of vehicular dynamics to obtain a reference generator that generates signals of a collision-free path which are to be tracked by a vehicle. A simple controller has been designed on the basis of this generator, therefore resulting in a dynamic controller ensuring obstacle avoidance. This technique can be applied for active vehicle control and in the case of autonomous guidance. Simulation results have been presented in the autonomous case considering a scenario inspired by the so-called moose (or elk) test, with the presence of other collaborative vehicles.

Future works will consist of developing a decisionmaking mechanism that will work at a higher level to assess threats and decide the best maneuver in the case of hazardous situations. Moreover, highly congested scenarios will be considered, which could cause the problem of local potential field minima, which could determine a block of the vehicle. Furthermore, the robustness of the proposed controller in the presence of measurement errors will be studied. Finally, a validation on software platforms, simulating the whole vehicle dynamics, will be performed.

\section{Conflicts of Interest}

The authors declare that they have no conflicts of interest.

\section{References}

[1] O. Khatib, "Real-time obstacle avoida nce for manipulators and mobile robots," International Journal of Robotics Research, vol. 5, no. 1, pp. 90-98, 1986.

[2] F. Arambula Cosío and M. A. Padilla Castañeda, "Autonomous robot navigation using adaptive potential fields," Mathematical and Computer Modelling, vol. 40, no. 9-10, pp. 1141-1156, 2004.

[3] W. H. Huang, B. R. Fajen, J. R. Fink, and W. H. Warren, "Visual navigation and obstacle avoidance using a steering potential function," Robotics and Autonomous Systems, vol. 54, no. 4, pp. 288-299, 2006.

[4] Y. Liu, Y. Zhao, and X. Zhou, "Design research on vehicle collision avoidance based on artificial potential field," Applied Mechanics and Materials, vol. 271, no. 1, pp. 727-731, 2013.

[5] R. Pepy, A. Lambert, and H. Mounier, "Path Planning Using a Dynamic Vehicle Model," Information and Communication Technologies, vol. 1, pp. 781-786, 2006.

[6] G. Roussos, D. V. Dimarogonas, and K. J. Kyriakopoulos, "3D navigation and collision avoidance for nonholonomic aircraftlike vehicles," International Journal of Adaptive Control and Signal Processing, vol. 24, no. 10, pp. 900-920, 2010. 
[7] M. Spenko, Y. Kuroda, S. Dubowsky, and K. Iagnemma, "Hazard avoidance for high-speed mobile robots in rough terrain," Journal of Field Robotics, vol. 23, no. 5, pp. 311-331, 2006.

[8] Y. Hattori, E. Ono, and S. Hosoe, "Optimum vehicle trajectory control for obstacle avoidance problem," IEEE/ASME Transactions on Mechatronics, vol. 11, no. 5, pp. 507-512, 2006.

[9] S. J. Anderson, S. C. Peters, T. E. Pilutti, and K. Iagnemma, "An optimal-control-based framework for trajectory planning, threat assessment, and semi-autonomous control of passenger vehicles in hazard avoidance Scenarios," International Journal of Vehicle Autonomous Systems, vol. 8, no. 2-4, pp. 190-216, 2010.

[10] Z. Qu, J. Wang, and C. E. Plaisted, "A new analytical solution to mobile robot trajectory generation in the presence of moving obstacles," IEEE Transactions on Robotics, vol. 20, no. 6, pp. 978993, 2004.

[11] L. Podsędkowski, J. Nowakowski, M. Idzikowski, and I. Vizvary, "New solution for path planning in partially known or unknown environment for nonholonomic mobile robots," Robotics and Autonomous Systems, vol. 34, no. 2-3, pp. 145-152, 2001.

[12] K. P. Tee, S. S. Ge, and E. H. Tay, "Barrier Lyapunov Functions for the control of output-constrained nonlinear systems," Automatica, vol. 45, no. 4, pp. 918-927, 2009.

[13] C. Acosta Lá, B. Castillo Toledo, R. Cespi, and S. Di Gennaro, "An integrated active nonlinear controller for wheeled vehicles," Journal of The Franklin Institute, vol. 352, no. 11, pp. 4890-4910, 2015.

[14] C. Pozna, F. Troester, R.-E. Precup, J. K. Tar, and S. Preitl, “On the design of an obstacle avoiding trajectory: method and simulation," Mathematics and Computers in Simulation, vol. 79, no. 7, pp. 2211-2226, 2009.

[15] J. C. Gerdes and E. J. Rossetter, "A unified approach to driver assistance systems based on artificial potential fields," Journal of Dynamic Systems, Measurement, and Control, vol. 123, no. 3, pp. 431-438, 2001.

[16] S. Shimoda, Y. Kuroda, and K. Iagnemma, "Potential field navigation of high speed unmanned ground vehicles on uneven terrain," in Proceedings of the 2005 IEEE International Conference on Robotics and Automation, pp. 2828-2833, April 2005.

[17] J. Ji, A. Khajepour, W. W. Melek, and Y. Huang, "Path planning and tracking for vehicle collision avoidance based on model predictive control with multiconstraints," IEEE Transactions on Vehicular Technology, vol. 66, no. 2, pp. 952-964, 2017.

[18] J. Y. Wong, Theory of Ground Vehicles, John Wiley \& Sons, 2008.

[19] G. Burgio and P. Zegelaar, "Integrated Vehicle Control Using Steering and Brakes," International Journal of Control, vol. 79, no. 2, pp. 162-169, 2006.

[20] R. Karbalaei, A. Ghaffari, R. Kazemi, and S. H. Tabatabaei, "Design of an integrated AFS/DYC based on fuzzy logic control," in Proceedings of the 2007 IEEE International Conference on Vehicular Electronics and Safety, ICVES, pp. 1-6, December 2007.

[21] S. Ç. Baslamisli, I. Polat, and I. E. Kose, "Gain scheduled active steering control based on a parametric bicycle model," in Proceedings of the 2007 IEEE Intelligent Vehicles Symposium, IV 2007, pp. 1168-1173, June 2007.

[22] J. Ackermann, J. Guldner, W. Sienel, R. Steinhauser, and V. I. Utkin, "Linear and Nonlinear Controller Design for Robust Automatic Steering," IEEE Transactions on Control Systems Technology, vol. 3, no. 1, pp. 132-143, 1995.
[23] S. Malan, M. Taragna, P. Borodani, and L. Gortan, "Robust performance design for a car steering device," in Proceedings of the 33rd IEEE Conference on Decision and Control. Part 1 (of 4), pp. 474-479, December 1994.

[24] H. B. Pacejka, Tyre and Vehicle Dynamics, Butterworth-Hein, Elsevier, 2005.

[25] E. J. Rossetter and J. C. Gerdes, "A Study of Lateral Vehicle Control Under a Virtual Force Framework," in Proceedings of the International Symposium on Advanced Vehicle Control - AVEC, pp. 9-13, 2002.

[26] C. Acosta Lúa and S. Di Gennaro, "Nonlinear adaptive tracking for ground vehicles in the presence of lateral wind disturbance and parameter variations," Journal of The Franklin Institute, vol. 354, no. 7, pp. 2742-2768, 2017.

[27] C. Acosta Lúa, S. Di Gennaro, and M. E. Sánchez Morales, "Nonlinear adaptive controller applied to an Antilock Braking System with parameters variations," International Journal of Control, Automation, and Systems, vol. 15, no. 5, pp. 2043-2052, 2017.

[28] A. Ukaew and C. Chauypen, "Implementation of Conceptual Real-Time Embedded Functional Design via Drive-By-Wire ECU Development," International Journal of Mechanical, Aerospace, Industrial, Mechatronic and Manufacturing Engineering, vol. 9, no. 6, pp. 924-930, 2015. 


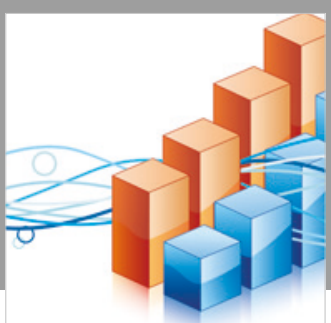

Advances in

Operations Research

\section{-n-m}
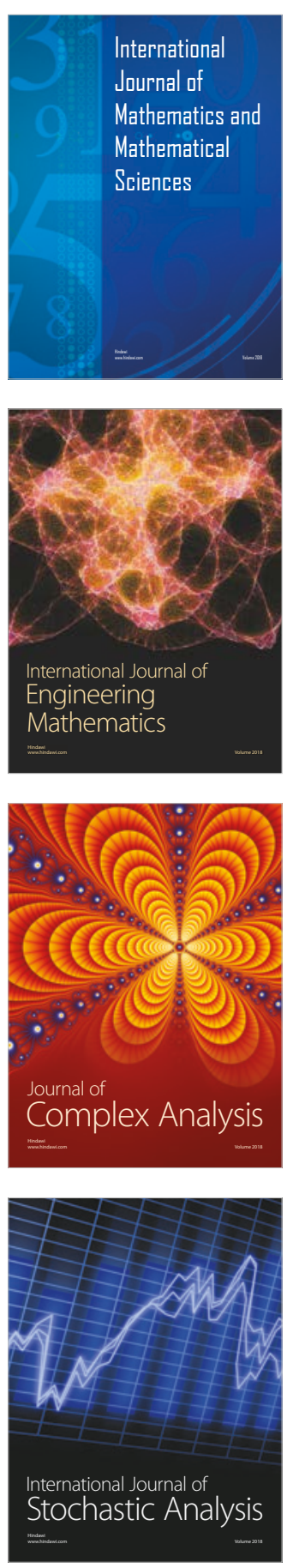
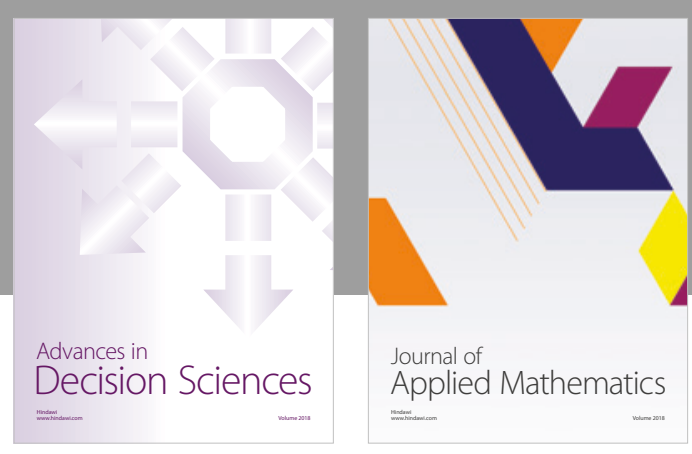

Journal of

Applied Mathematics
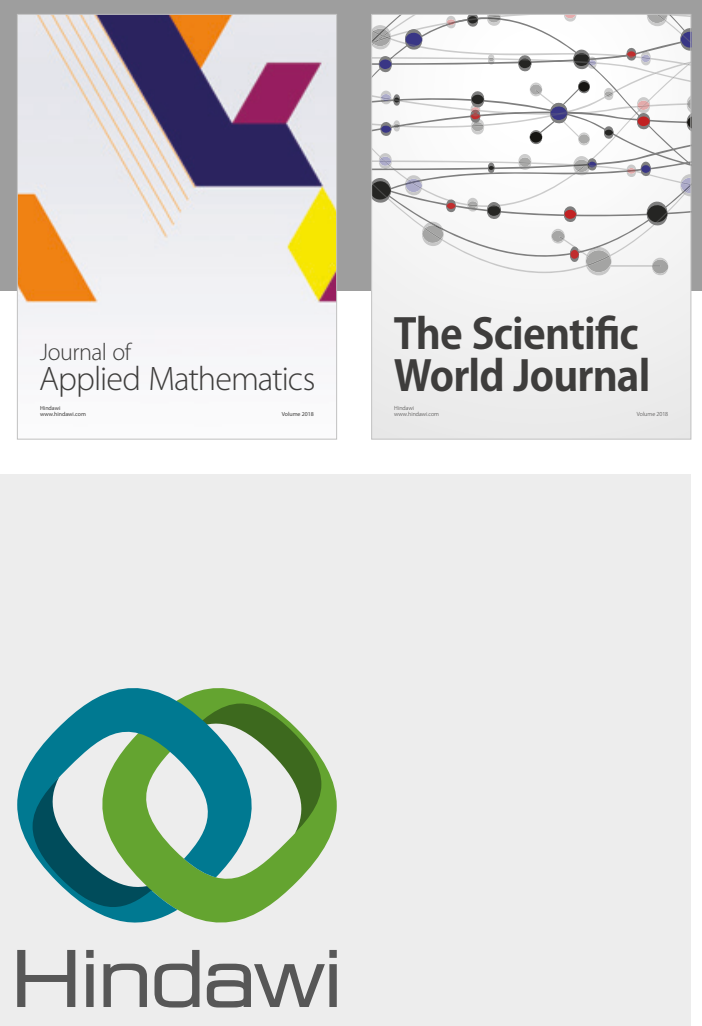

Submit your manuscripts at

www.hindawi.com

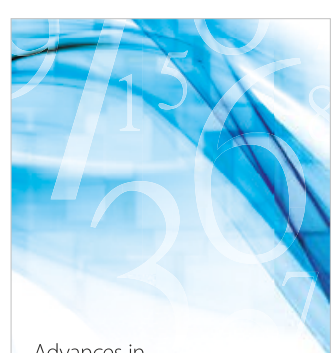

Advances in
Numerical Analysis
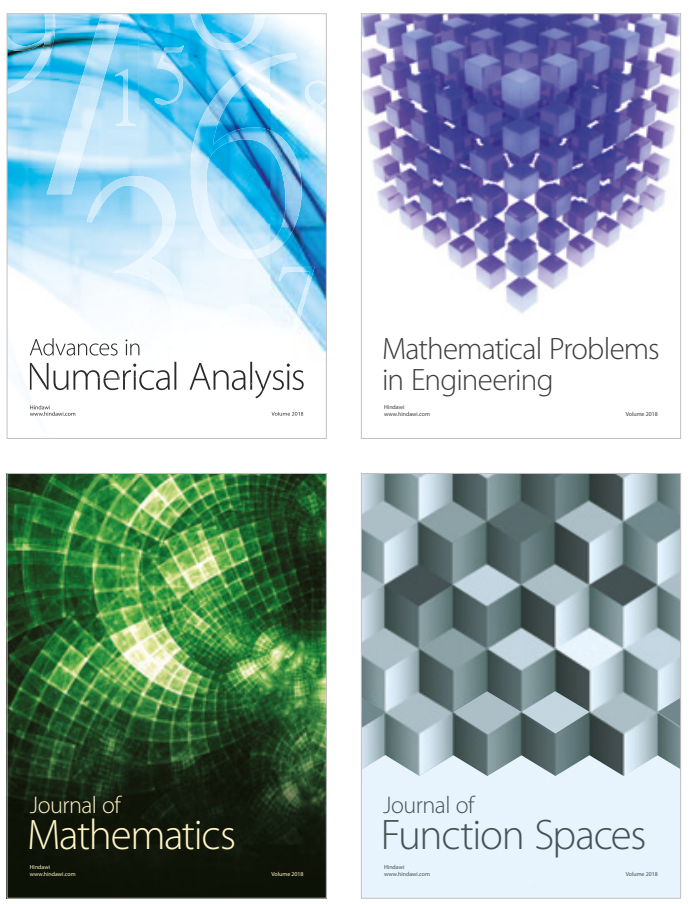

Mathematical Problems in Engineering

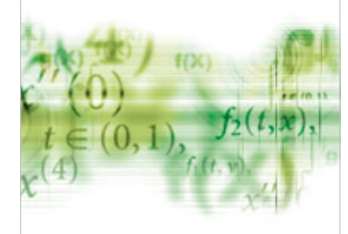

International Journal of

Differential Equations

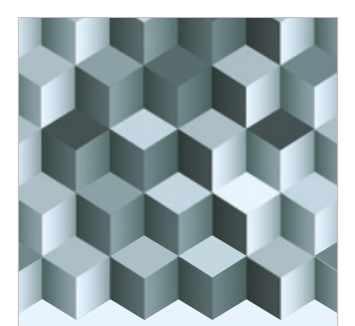

Journal of

Function Spaces

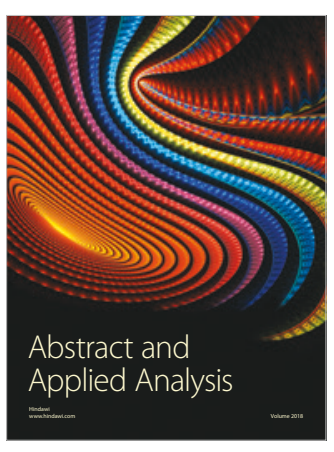

The Scientific

World Journal

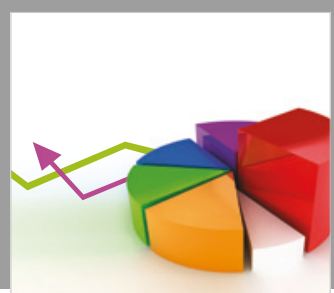

Journal of

Probability and Statistics
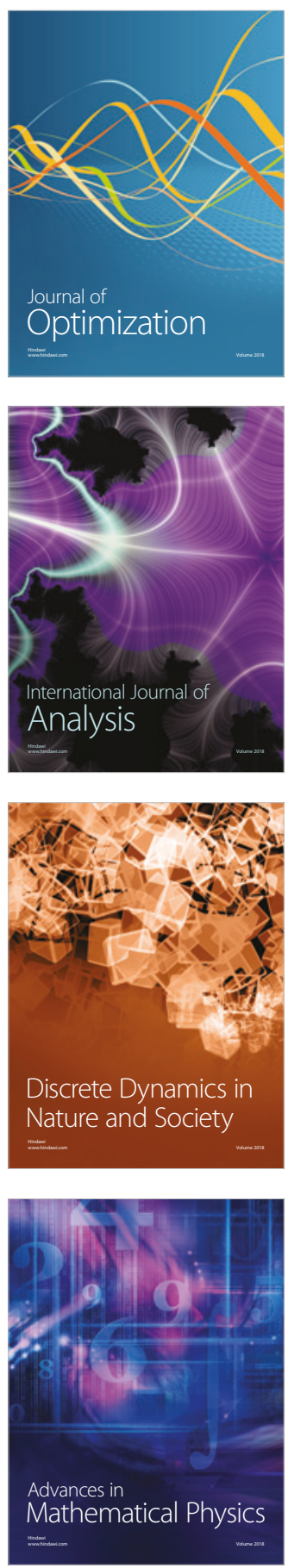